\title{
How to identify gene-environment interactions in a multifactorial disease: CHD as an example
}

\author{
Philippa J. Talmud \\ Centre for Cardiovascular Genetics, British Heart Foundation Laboratories, Rayne Building, \\ Royal Free and University College London Medical School, London WC1E 6JF, UK
}

\begin{abstract}
CHD is a multifactorial disease, caused by both genetic and environmental factors. The inherited 'defective' genes will vary from individual to individual, and any single mutation is likely to be making only a small contribution to risk. The context dependency, i.e. the importance of environmental factors in influencing genetic risk, is now becoming evident. Thus, a mutation may have a modest effect on risk in individuals who maintain a low environmental risk, but a major effect in a high-risk environment. Methods of analysing gene-environment interactions on CHD risk will be discussed and illustrated with several examples. $A P O E$ has three common alleles, $\varepsilon 2, \varepsilon 3$ and $\varepsilon 4$. The $\varepsilon 4$ allele has consistently been associated with CHD risk, which has been confirmed by meta-analysis. However, when the effect of genotype on risk was considered in smokers and non-smokers separately, risk in non-smokers was similar in all $A P O E$ genotypes. By comparison, in the smokers, $\varepsilon 3$ homozygotes, as expected, had an approximately 2 -fold higher risk, while for $\varepsilon 4$ carriers there was a significantly greater than additive effect of genotype and smoking on risk $(P<0 \cdot 007)$. Thus, the impact of the $\varepsilon 4$ allele on CHD risk appears to be confined to current smokers, an effect that has been confirmed in several studies. Another example is the interaction between the alcohol dehydrogenase 3 gene variant and alcohol consumption on CHD risk $(P<0 \cdot 001)$, showing the context dependency of the effect. Thus, the importance of considering environmental factors as potential genotype-risk modifiers has major public health implications.
\end{abstract}

Gene-environment interaction: $A P O E$ : $A D H 3$ : Diet: Alcohol consumption: Smoking

\section{CHD: an example of a complex disorder}

CHD is the major cause of mortality in the Western world and the incidence in the Far East, where prevalence until recently has been low, is rising at disturbing levels. Ethnic or racial difference may account for some of the disparity in mortality rate between the East and the West. However, the recent increase in CHD mortality in Japan and China, for example, must reflect lifestyle changes, since genetic predisposition is stable over many generations. Like most complex diseases of late onset, CHD may be thought of as a failure of adequate homeostasis within a physiological system. This failure may arise as a result of variation at the genetic level (e.g. gene transcription or altered protein function) or environmental exposure (e.g. diet, cigarette consumption etc.), but in practice is a result of the interaction between genetic and environmental factors. Rarely does CHD arise from a single mutation in a single gene. The most relevant example of monogenic CHD is familial hypercholesterolaemia, which occurs in approximately one in 500 members of the population (Goldstein \& Brown, 1989) and results from mutations in one of three genes, the LDL receptor gene (Goldstein \& Brown, 1989), the $A P O B$ gene (Innerarity et al. 1990) and the recentlyidentified PCSK 9 gene that encodes proprotein convertase subtilisin/kexin type 9 (Abifadel et al. 2003), all eliciting the same phenotype. More commonly, an individual with CHD will have inherited minor functional mutations or gene variants in several different genes coding for key proteins that are involved in maintaining cardiovascular health. These combined genetic effects account for much of the biological diversity in homeostatic systems, and in their absence all human subjects would respond in an identical manner to an environmental challenge. In reality, 
(a)

Environment effect, no genotype effect

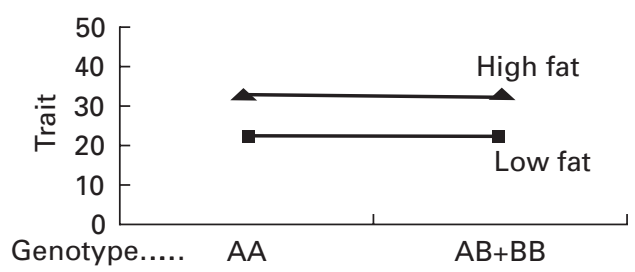

(c) Environment effect, genotype effect: no interaction

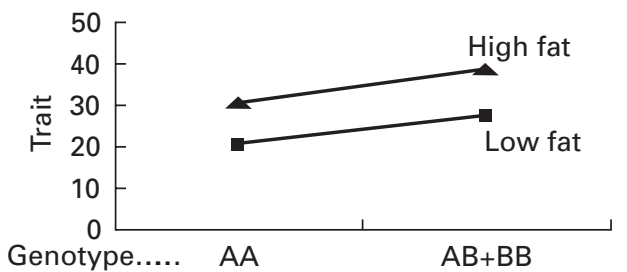

(b) Genotype effect, no environment effect

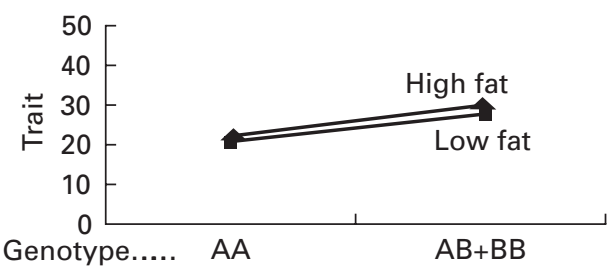

(d) Environment effect, genotype effect: significant interaction

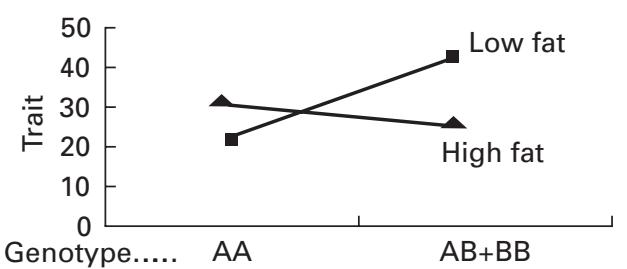

Fig. 1. Four options to illustrate gene-environment interaction: (a) genotype ( $A A$ compared with $A B+B B$ ) is not having an effect on the quantitative trait but the change in environment (e.g. from high-fat diet to low-fat diet) is affecting the trait; (b) environment is not having an effect on the quantitative trait, but genotype is; (c) both genotype and environment are affecting the trait but the effect of environment is the same in both genotypes so there is no interaction; (d) both environment and genotype are affecting the trait but in this case the interaction would be statistically significant, deviating from additive.

this is not the case. For example, some individuals exposed to cigarette smoke with an otherwise identical CHD risk profile, e.g. plasma cholesterol, will go on to develop the disease whilst others will not. Thus, the interaction of functional gene polymorphisms with environmental factors (gene-environment interactions) plays a substantial role in risk.

\section{Gene-environment interaction and how to analyse the data}

Gene-environment interaction implies that in combination the effect of genotype and environment deviates from the additive or multiplicative effects of the two factors. The situation is illustrated in Fig. 1. However, many studies have simply corrected for traditional CHD risk factors such as smoking, cholesterol, blood pressure etc. in the statistical analysis instead of investigating whether there is an interaction.

A simple statistical approach to the analysis of geneenvironment interaction when dealing with a quantitative trait (e.g. blood pressure) is to use the statistical test analysis of covariance, with the quantitative trait entered as the dependent variable, genotype and environmental factors entered as main effects and with an interaction term between the genotypic and environmental factor. If this term is statistically significant the implication is that there is a greater (or lesser) than additive effect and interaction is suggested. However, this outcome could be a result of chance alone, which highlights the importance of confirmatory studies. If the $P$ value for the interactive term is not significant the implication is that there is a lack of interaction, but it could also simply reflect the lack of
Table 1. How to test for interaction of environment (e.g. smoking) and genotype on a quantitative trait (e.g. blood pressure (BP)) and a binary trait (e.g. CHD risk)

\begin{tabular}{lcccc}
\hline Environment & Genotype & Trait BP $(\mathrm{mmHg})$ & Trait BP $(\mathrm{mmHg})$ \\
\hline Smoking: & $\mathrm{N}$ & $\mathrm{AA}$ & 100 & 100 \\
& $\mathrm{Y}$ & $\mathrm{AA}$ & 130 & 130 \\
$\mathrm{~N}$ & $\mathrm{AB}+\mathrm{BB}$ & 120 & 120 \\
$\mathrm{Y}$ & $\mathrm{AB}+\mathrm{BB}$ & 150 & 160 \\
& & & No interaction: & Interaction, \\
& & & additive & deviation from \\
& & & & additive \\
High-fat diet: & $\mathrm{N}$ & 11 & OR for CHD & OR for CHD \\
& $\mathrm{Y}$ & 11 & $1 \cdot 00$ & $1 \cdot 00$ \\
& $\mathrm{~N}$ & $12+22$ & $2 \cdot 00$ & $2 \cdot 00$ \\
& $\mathrm{Y}$ & $12+22$ & $3 \cdot 00$ & $3 \cdot 00$ \\
& & & No interaction: & $7 \cdot 50$ \\
& & & multiplicative & Interaction, \\
& & & & deviation from \\
& & & & multiplicative \\
\hline
\end{tabular}

$\mathrm{N}$, no; $\mathrm{Y}$, yes; OR, odds ratio.

power in the study to detect an effect (Table 1). For a binary trait, e.g. deep-vein thrombosis, logistic regression or survival analyses are used to look for interaction. Here a significant $P$ value implies greater than multiplicative interaction, with the same provisos of lack of power for non-statistical effects and chance for statistically significant outcome (Table 1).

From a mechanistic viewpoint interaction suggests that at the molecular level the effect or byproduct of the environmental insult modifies the molecular function of the product of the gene under observation. 


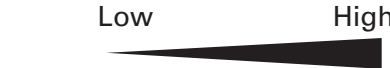

Genes of small effect: ranging from few to many
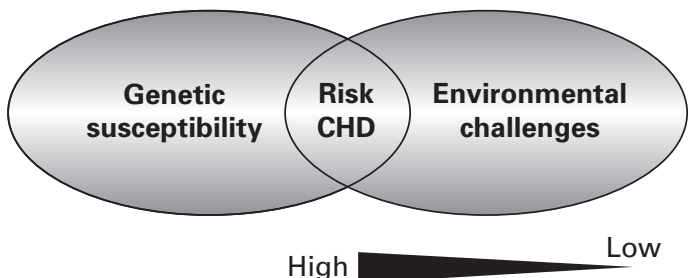

Different lifestyles: ranging from healthy to unhealthy

Fig. 2. Model for gene-environment interaction. This model proposes that each individual occupies a position on the genetic risk spectrum, depending on how many risk-increasing gene variants have been inherited. Similarly, individuals are exposed to a range of environmental challenges depending on lifestyle choices. It is proposed that risk of $\mathrm{CHD}$ only occurs when an individual at high genetic risk enters a high-risk environment, and that the genetic risks and environment risks alone will not trigger a $\mathrm{CHD}$ event.

A working model for the interaction of genetic and environmental factors is shown in Fig. 2. The hypothesis is that in the population there is a range of genetic risk profiles, with each individual occupying a position along the risk spectrum from a low to a high genetic risk depending on the number of 'defective' alleles at key loci that they have inherited. Individuals adopt a different position on the environmental spectrum of risk by the lifestyle choices they make (e.g. low-fat diet and non-smokers to high-fat diet and smokers). Thus, while the environmental risk factors are modifiable the genetic risk factors are not. The importance of gene-environment interaction is that only when an individual with a high-risk genetic profile enters a high-risk environment will the effect on risk be so great that premature CHD develops. Understanding this gene-environment relationship is one of the big challenges that is being faced. The present review focuses on some recent reports of common genetic variants that are associated with significant effects on CHD risk lipid traits or excess CHD risk when individuals are exposed to a 'high-risk' environment.

\section{Statistical interaction $v$. biological interaction}

It is important not to confuse statistical and biological interaction. Although they both refer to the term 'interaction', they are very different.

Statistical interaction is a measure of association heterogeneity, and implies that the statistical description deviates from the expected joint effects, be they additive or multiplicative. Statistical interaction also depends on the statistical test used, e.g. statistical interaction might be evident for risk differences but not risk ratios. Take, for example, a purely hypothetical study looking at the effect of the Factor V Leiden mutation and oral contraception on the risk of deep-vein thrombosis in women. If for example, in the group on oral contraception the relative risk was found to be 12 for those carrying the Factor V Leiden mutation and 6 for those without the mutation, then the risk ratio would be $2(12 / 6)$. If the relative risk for the women not on oral contraception, but carrying the Factor $\mathrm{V}$ Leiden mutation was found to be 4 compared with 2 for the non-carriers, then the risk ratio is also $2(4: 2)$ and not different from that for those on contraception. If, however, the risk differences were compared, the value would be 6 $(12-6)$ for the group on oral contraception but only 2 $(4-2)$ for the group not on oral contraception, implying interaction. Thus, the statistical test is crucial for identifying interaction.

Biological interaction, or causal interaction, is the interdependent operation of two or more factors to produce or prevent an effect. Since every disease is measured by multiple components acting together, any cause is impinged on by other causes. An example of this situation is the association between smoking and lung cancer, where only a proportion of smokers develop cancer. The reason for this outcome is that other environmental or genetic factors modulate the effect on smoking. Thus, the outcome can be considered to be the interaction of smoking and other factors on cancer susceptibility. In fact, biological interactions are the basis of all complex diseases, whether they are a result of gene-gene or gene-environment interactions.

\section{Measuring genetic and environmental factors}

\section{Environmental risk factors}

There are several well-documented environmental variables that influence plasma lipid levels and increase CHD risk. These variables include dietary fat (for example, see Ginsberg et al. 1998), smoking (for example, see Doll \& Hill, 1966), alcohol consumption (for review, see Savolainen \& Kesaniemi, 1995) and low exercise (for example, see Haskell, 1986). However, these environmental variables are very difficult to quantify with accuracy in free-living individuals. In small dietary intervention studies or control exercise studies it is possible to estimate energy intake and expenditure with good precision, but such studies have very little power for genetic studies, or for examining gene-environment interactions. Most environmental factors are assessed by questionnaire and may be under-reported, e.g. smoking or alcohol consumption, or over-reported in the case of exercise. In addition, it is hard to adjust for factors such as the tar content of cigarettes (Shields, 2000), which might alter the impact of smoking. With food consumption daily food records may be useful, but assessing the fat content and fatty acid composition of food is not straightforward. What is really needed are biomarkers for environmental factors, i.e. plasma measures of byproducts that can be quantified, such as plasma cotinine as a measure of cigarette consumption or levels of plasma linoleic acid as a measure of dietary compliance (Wallace et al. 2000).

\section{Genetic risk factors}

Easy access to the human genome databases has improved the availability of genetic information, and thus designing assays for genotyping new and variant sites in candidate 
genes is easier than ever. High-throughput genotyping methodology has also advanced considerably and the ability to genotype large studies at several sites is becoming more straightforward. Genotype error rates are also low, and it has been estimated for a case-control study that genotyping twenty-five individuals would be sufficient to give an approximately unbiased estimate of genotype errors (Rice \& Holmans, 2003).

\section{The impact of smoking on lipid levels and CHD risk}

Of the environmental factors studied, smoking is perhaps the easiest and most commonly analysed. Smoking is known to approximately double lifetime risk of CHD (Doll \& Hill, 1966), and is thought to increase cardiovascular risk by several different mechanisms, i.e. by directly damaging vascular endothelium, leading to increased secretion of adhesion molecules, and by perturbing lipoprotein metabolism and increasing insulin resistance and lipid intolerance. In addition, smoking-induced lung and endothelial wall damage will lead to an IL-6-mediated inflammatory response, causing hepatic up-regulation of fibrinogen expression and increased risk of thrombosis. As a result of dietary habits, smokers also have lower levels of antioxidants such as ascorbate and tocopherol, and thus smoking would favour the oxidation of LDL (Fickl et al. 1996) and increase the risk of atherosclerosis.

\section{APOE: smoking interaction and CHD risk}

$A P O E$ with its common variant alleles, $\varepsilon 2$, $\varepsilon 3$ and $\varepsilon 4$, has been the subject of intense interest because of the reported predisposition of $\varepsilon 4$ carriers $(\varepsilon 4+)$ to both early onset of Alzheimer's disease (Corder et al. 1993; Poirier et al. 1993) and CHD. A meta-analysis assessing the impact of $A P O E$ variants on CHD risk (Wilson et al. 1996) considered fourteen published studies, primarily with a case-control design, examining both clinical CHD and angiographically-defined CHD. Compared with $\varepsilon 3$ homozygotes the summary estimates of the odds ratios on CHD events for both sexes combined showed $\varepsilon 4$ to have an odds ratio of $1.26(95 \%$ CI $1.13,1.41)$, which was similar to that for both men and women when considered separately. However, none of these studies considered environmental factors as potential genotype-risk modifiers, but simply corrected for non-lipid risk factors, amongst them smoking, when examining the risk association between $A P O E$ genotype and CHD.

So, is there evidence for the context dependency of $A P O E$ genotype on risk? The effect of smoking and $A P O E$ genotype on CHD risk was examined in the UK-based Northwick Park Heart Study of $>3000$ men followed prospectively for CHD events for $>6$ years (Humphries et al. 2001). The relative risk of smoking alone on CHD risk (expressed as a hazard ratio) was, as expected, 1.94 (95\% CI $1 \cdot 25,3 \cdot 01)$. APOE genotype was associated with effects on cholesterol and apoB levels, with $\varepsilon 4$ carriers having the highest levels and $\varepsilon 2$ carriers the lowest levels, irrespective of smoking status. Compared with all the nonsmokers, for whom the hazard ratio was set at $1 \cdot 00$, of the men who smoked those with the genotype $\varepsilon 3 / \varepsilon 3$ had a hazard ratio of $1.68(95 \%$ CI $1 \cdot 01,2 \cdot 83)$ compared with $1 \cdot 18(95 \%$ CI $0 \cdot 46,3.03)$ for $\varepsilon 2$ carriers and $3 \cdot 17(95 \%$ CI $1.82,5.51)$ for $\varepsilon 4$ carriers. The interaction between smoking status and $A P O E$ genotype on CHD risk was significant $(P=0 \cdot 007)$ and was independent of BMI, blood pressure, lipid levels and markers of inflammation. Risk in men with the $\varepsilon 4$ allele who had quit smoking before the study began was lower, and did not differ from that of never-smokers, emphasising the benefit of smoking cessation. There is good evidence that $A P O E$ genotype influences lesion formation independently of its effects on fasting plasma lipid levels. The interaction between the $\varepsilon 4$ allele and smoking suggests that smoking exacerbates this effect. Although a recent large case-control study claimed to refute this high $A P O E$ \&4-smoking interaction on risk (Keaveny et al. 2003), our re-analysis of this data suggests risk is indeed higher in $\varepsilon 4$ carriers who smoke, showing a greater than additive effect and supporting data from the Northwick Park Heart Study (Humphries et al. 2003).

The most likely mechanism to explain the $\varepsilon 4$ allelesmoking interaction on CHD risk is through a direct effect on LDL oxidation. Several studies using recombinant apoE have demonstrated that the protection against oxidation in vitro is apoE2 $>$ apoE3 $>$ apoE4 (Smith et al. 1998). This effect may be because apoE2 has two free SH groups, apoE3 has one and apoE4 none, or may be due to other effects of apoE isoforms on the physico-chemical properties of lipoproteins that promote or protect from oxidation. The differential oxidation of apoE isoforms has now been confirmed in vitro with, as expected, apoE4 being more susceptible to oxidation than apoE3, which in turn is more susceptible than apoE2 (Jolivalt et al. 2000).

\section{Alcohol consumption, alcohol dehydrogenase gene and CHD risk}

Another example of the context dependency of a genetic variant on risk of CHD is the way in which the beneficial effect of moderate alcohol consumption is modified by variation in the gene for alcohol dehydrogenase (ADH). Moderate alcohol consumption has been shown to be cardio-protective, with risk following a ' $\mathrm{J}$ '-shaped curve (Doll et al. 1997), such that individuals with a low alcohol intake are at greater risk than those who consume moderate amounts of alcohol, but as alcohol intake increases, the risk of CHD increases. ADH is an isoenzyme that oxidises ethanol. It comprises three subunits, ADH1, 2 and 3. Genetic variation in AHD3 is common and is the best studied of the three genes. While the $A D H 3 \gamma 1$ allele leads to rapid oxidation of ethanol, the $\gamma 2$ allele results in a $2 \cdot 5$-fold slower rate of oxidation (Bosron et al. 1988). Hines et al. (2001) showed in an all-male nested casecontrol study drawn from the Physicians Health Study (and confirmed in other studies) that moderate alcohol consumption of one drink daily was associated, as expected, with a decreased risk of myocardial infarction. In the same study they also showed that the $A D H 3$ genotype was also associated with a difference in CHD risk. The question under investigation was whether alcohol intake modifies the genotypic effects on CHD risk. In those men who 
drank less than one drink per d (low alcohol consumers) there was no difference in the relative risk of a myocardial infarction in $\gamma 2$ carriers compared with $\gamma 1$-homozygous men. However, in the group who consumed one or more drinks daily the relative risk for the $\gamma 1 \gamma 1$ moderate drinkers when compared with the $\gamma 1 \gamma 1$ low alcohol consumers was $0.62(95 \%$ CI $0.34,1.132)$, while the relative risk for the $\gamma 2 \gamma 2$ moderate drinkers compared with $\gamma 2 \gamma 2$ low alcohol consumers was only $0 \cdot 14(95 \%$ CI $0 \cdot 04,0 \cdot 45)$. Thus, without taking both genotype and environment into account an accurate relative risk would not be obtained. However, in part the risk reduction was explained by a larger increase in HDL levels in the $\gamma 2 \gamma 2$ men $(P=0.01$ for interaction), and to what extent this genotype effect could be simply estimated by measures of habitual HDL levels is unclear.

\section{Conclusion}

Thus, the interaction between $A P O E$ and smoking and $A D H 3$ and alcohol consumption on CHD risk are analogous. In the studies described earlier genotype, without taking environment into account, was having an effect on risk, with $\varepsilon 4$ carriers having a greater risk than \&3 homozygotes and $A D H 3 \gamma 2$ homozygotes having a protective effect on risk compared with $\gamma 1$ homozygotes. However, when environment was included in the analysis rather than corrected for, and an interaction term was included in the statistical analyses, the results show that $A P O E$ \&4 genotype and $A D H 3 \gamma 2$ genotype do not behave uniformly. In non-smoking $A P O E$ \&4 carriers there was no effect of genotype on risk, similarly in low-alcoholconsuming $A D H 3 \gamma 2$ carriers CHD risk was not significantly different from that for $\gamma 1$ homozygotes. However, in $A P O E$ $\varepsilon 4$ carriers who smoked there was an increased CHD risk compared with common allele homozygotes who did not smoke, while $A D H 3 \gamma 2$ homozygotes with a moderate alcohol consumption were protected from risk when compared with the reference group.

Gene-environment interaction therefore provides additional insight into the pathophysiology of disease. From a public health viewpoint this factor has great implications, because it emphasises the reduced risk in never-smokers and ex-smokers and promotes the idea of smoking cessation. Similarly, it promotes the benefits of moderate alcohol consumption in all individuals, with a particular benefit in a genotype-specific manner.

So how is the field expected to progress over the next 3-5 years? Concern has been raised about the apparent 'inconsistency' of genetic association studies, with genotypes being associated with large effects on CHD risk in the initial published report (often in high-impact-factor journals, but on relatively small samples), but with subsequent (larger) studies failing to confirm this effect, or at best finding an effect of much more modest size. Luckily, this problem is amenable to meta-analysis, and such an analysis of thirty-six associations in CHD, diabetes, hypertension, cancer etc. was recently published (Ioannidis et al. 2001). Not surprisingly, the major discriminating factor in predicting whether an initial risk association was subsequently confirmed was the size of the original sample.
Even though the results of such a meta-analysis are confined to published studies, and the difficulty in publishing 'negative' studies raises concerns about publication bias, the overall message of this paper is extremely encouraging, i.e. that many of the reported associations between certain genotypes and disease risk do stand up to the test of replication.

The same sort of rules must be applied to the geneenvironment interactions. It is obviously worth publishing such interactions, providing they are carried out on a reasonably-sized sample, because they will stimulate workers in the field to try to replicate the observation. It should be recognised, however, that where a particular genotype is only associated with risk in the presence of a particular environment (e.g. high-fat diet, cigarette smoking etc.), the failure to observe an association between this genotype and risk in a second sample may be explained by the relatively lower prevalence of the important environmental factor. Clearly, there is still a lot of work to be done in order to tease out the genetic factors involved in CHD risk in the general population.

\section{References}

Abifadel M, Varret M, Rabes JP, Allard D, Ouguerram K, Devillers M et al. (2003) Mutations in PCSK9 cause autosomal dominant hypercholesterolemia. Nature Genetics 34, 154-156.

Bosron WF, Lumeng L \& Li TK (1988) Genetic polymorphism of enzymes of alcohol metabolism and susceptibility to alcoholic liver disease. Molecular Aspects of Medicine 10, 147-158.

Corder EH, Saunders AM, Strittmatter WJ, Schmechel DE, Gaskell PC, Small GW, Roses AD, Haines JL \& PericakVance MA (1993) Gene dose of apolipoprotein E type 4 allele and the risk of Alzheimer's disease in late onset families. Science 261, 921-923.

Doll R \& Hill AB (1966) Mortality of British doctors in relation to smoking; observation on coronary thrombosis. National Cancer Institute Monographs 19, 205-268.

Doll R, Peto R, Hall E, Wheatley K \& Gray R (1997) Alcohol and coronary heart disease reduction among British doctors: confounding or causality? European Heart Journal 18, 23-25.

Fickl H, Van Antwerpen VL, Richards GA, Van der Westhuyzen DR, Davies N, Van der Walt R, Van der Merwe CA \& Anderson R (1996) Increased levels of autoantibodies to cardiolipin and oxidised low density lipoprotein are inversely associated with plasma vitamin $\mathrm{C}$ status in cigarette smokers. Atherosclerosis 124, 75-81.

Ginsberg HN, Kris-Etherton P, Dennis B, Elmer PJ, Ershow A, Lefevre M, et al. (1998) Effects of reducing dietary saturated fatty acids on plasma lipids and lipoproteins in healthy subjects: the DELTA Study, protocol 1. Arteriosclerosis, Thrombosis and Vascular Biology 18, 441-449.

Goldstein JL \& Brown MS (1989) Familial hypercholesterolemia. In The Metabolic Basis of Inherited Disease, 6th ed., pp. 1215-1250 [CH Scriver, AI Beaudet, WS Sly and D Valle, editors]. New York: McGraw-Hill Book Co.

Haskell WL (1986) The influence of exercise training on plasma lipids and lipoproteins in health and disease. Acta Medica Scandinavica 711, Suppl., 25-37.

Hines LM, Stampfer MJ, Ma J, Gaziano JM, Ridker PM, Hankinson SE, Sacks F, Rimm EB \& Hunter DJ (2001) Genetic variation in alcohol dehydrogenase and the beneficial 
effect of moderate alcohol consumption on myocardial infarction. New England Journal of Medicine 344, 549-555.

Humphries SE, Hawe E, Dhamrait S, Miller GJ \& Talmud PJ (2003) In search of genetic precision. Lancet 361, 1908-1909.

Humphries SE, Talmud PJ, Hawe E, Bolla M, Day IN \& Miller GJ (2001) Apolipoprotein E4 and coronary heart disease in middle-aged men who smoke: a prospective study. Lancet $\mathbf{3 5 8}$, $115-119$.

Innerarity TL, Mahley RW, Weisgraber KH, Bersot TP, Krauss RM, Vega GL, Grundy SM, Friedl W, Davignon J \& McCarthy BJ (1990) Familial defective apolipoprotein B-100: a mutation of apolipoprotein B that causes hypercholesterolemia. Journal of Lipid Research 31, 1337-1349.

Ioannidis JP, Ntzani EE, Trikalinos TA \& Contopoulos-Ioannidis DG (2001) Replication validity of genetic association studies. Nature Genetics 29, 306-309.

Jolivalt C, Leininger-Muller B, Bertrand P, Herber R, Christen Y \& Siest G (2000) Differential oxidation of apolipoprotein E isoforms and interaction with phospholipids. Free Radical Biology in Medicine 28, 129-140.

Keavney B, Parish S, Palmer A, Clark S, Youngman L, Danesh J, McKenzie C, Delepine M, Lathrop M, Peto R \& Collins R (2003) Large-scale evidence that the cardiotoxicity of smoking is not significantly modified by the apolipoprotein E epsilon 2/epsilon 3/epsilon 4 genotype. Lancet 361, 396-398.
Poirier J, Davignon J, Bouthillier D, Kogan S, Bertrand P \& Gauthier S (1993) Apolipoprotein E polymorphism and Alzheimer's disease. Lancet 342, 697-699.

Rice KM \& Holmans P (2003) Allowing for genotyping error in analysis of unmatched case-control studies. Annals of Human Genetics 67, 165-174.

Savolainen MJ \& Kesaniemi YA (1995) Effects of alcohol on lipoproteins in relation to coronary heart disease. Current Opinion in Lipidology 6, 243-250.

Shields PG (2000) Epidemiology of tobacco carcinogenesis. Current Oncology Reports 2, 257-262.

Smith JD, Miyata M, Poulin SE, Neveux LM \& Craig WY (1998) The relationship between apolipoprotein $\mathrm{E}$ and serum oxidation-related variables is apolipoprotein E phenotype dependent. International Journal of Clinical Laboratory Research 28, 116-121.

Wallace AJ, Mann JI, Sutherland WH, Williams S, Chisholm A, Skeaff CM, Gudnason V, Talmud PJ \& Humphries SE (2000) Variants in the cholesterol ester transfer protein and lipoprotein lipase genes are predictors of plasma cholesterol response to dietary change. Atherosclerosis 152, 327-336.

Wilson PW, Schaefer EJ, Larson MG \& Ordovas JM (1996) Apolipoprotein E alleles and risk of coronary disease. A metaanalysis. Arteriosclerosis, Thrombosis and Vascular Biology 16, 1250-1255. 